\title{
The Impact of Comorbidity on Patient-Reported Outcomes in Psoriatic Arthritis: A Systematic Literature Review
}

\author{
Juan D. Cañete · Jose Antonio Pinto Tasende - Francisco José Rebollo Laserna • \\ Susana Gómez Castro · Rubén Queiro
}

Received: February 12, 2020 / Published online: April 8, 2020

(C) The Author(s) 2020

\begin{abstract}
Introduction: A systematic literature review was conducted with the aim to analyse the impact of comorbidity on patient-reported outcomes (PROs) in patients with psoriatic arthritis (PsA).

Methods: A sensitive search strategy of the Medline, Embase and the Cochrane Library (up to March 2019) was applied to retrieve studies for inclusion in this systematic literature review.
\end{abstract}

Enhanced Digital Features To view enhanced digital features for this article go to: https://doi.org/10.6084/ m9.figshare.12002604.

Electronic Supplementary Material The online version of this article (https://doi.org/10.1007/s40744020-00202-x) contains supplementary material, which is available to authorized users.

J. D. Cañete

Arthritis Unit, Rheumatology Department, Hospital Clínic-IDIBAPS, Barcelona, Spain

J. A. P. Tasende

Rheumatology Department, Complexo Hospitalario Universitario de A Coruña-INIBIC, A Coruña, Spain

F. J. R. Laserna · S. G. Castro

Pfizer Medical Department, Alcobendas, Madrid, Spain

\section{R. Queiro (ه)}

Rheumatology Department, Hospital Universitario Central de Asturias, Oviedo, Spain

e-mail: rubenque7@yahoo.es
Abstracts of the ACR and EULAR scientific meetings were also searched. The selection criteria were: (1) patients with PsA (population) with a comorbidity (intervention) and (2) report of any impact of the comorbidity on PROs. Systematic literature reviews, randomized controlled trials and observational were included in this systematic literature review. Two of the authors selected the articles and collected the data.

Results: Eighteen articles were included in this systematic literature review, with most being cross-sectional studies that included more than 9000 patients with PsA. Some studies analysed the impact of an individual comorbidity, such as fibromyalgia (FM), and in others the analysis was according to the number of comorbidities. The most frequently analysed PROs were function, quality of life and fatigue. Analysis of the studies included in the review showed that patients with a higher number of comorbidities and/or more severe comorbidities reported worse impacts of their disease on function, patient's global assessment (PGA), pain, fatigue, work disability and quality of life. Specifically, FM had a negative impact on the Bath Ankylosing Spondylitis Disease Activity Index (BASDAI), function, quality of sleep and quality of life; anxiety and depression had a negative impact on function and fatigue; metabolic syndrome had a negative impact on BASDAI, function, PGA and quality of life; obesity had a negative impact on function and pain; smoking 
(current and ex-smokers) had a negative impact on pain, function, fatigue, quality of life and overall health; alcohol intake had a negative impact on pain, function, fatigue, quality of life and overall health.

Conclusions: The prevalence and impact of medical comorbidity on PROs are very high in patients with PsA.

Keywords: Comorbidity; Patient-reported outcomes; Psoriatic arthritis; Systematic literature review

\section{Key Summary Points}

More than half of patients with psoriatic arthritis (PsA) have at least 1 comorbidity.

Comorbidity has several impacts at different levels in PsA, including patientreported outcomes (PROs).

PsA patients with more number or severity of comorbidities reported a worse impact on: function, patient's global assessment (PGA), pain, fatigue, work disability and quality of life.

In PsA the prevalence and impact of comorbidity on PROs are very high.

\section{INTRODUCTION}

Psoriatic arthritis (PsA) is a chronic inflammatory disease with an incidence and prevalence ranging from approximately 3-23 persons per 100,000 and $0.5-4$ persons per 1000, respectively, in Western countries [1]. It has been reported that more than half of patients with PsA have at least one comorbidity [2, 3]. The most prevalent comorbidities associated with PsA are cardiovascular disease, metabolic syndrome, depression and anxiety, osteoporosis, ophthalmic, liver, kidney and inflammatory bowel disease $[2,4,5]$.

The impact of comorbidity on PSA patients can be described at different levels [6-8]. The increased mortality risk observed in PsA patients is mainly explained by the high prevalence of these comorbidities, especially cardiovascular disease [6]. Similarly, the presence of comorbidities may also have an impact on the activity and/ or the severity outcomes measures of PsA. For example, fibromyalgia (FM) or depression can considerably influence the score of the Bath Ankylosing Spondylitis Disease Activity Index (BASDAI) in PsA patients [9]. It has also been reported that the presence of comorbidities may influence the treatment response and decisionmaking process. Different studies have shown that both obesity and metabolic syndrome are associated with lower retention rates of and response rates to biological therapy [10-12], and a systematic literature review found that obesity and overweight reduce the odds to achieve minimal disease activity in patients with PsA receiving treatment with traditional or biologic drugs [13].

Comorbidity can also result in greater functional impairment and have an important negative impact on the quality of life [14]. These outcomes are included in the patient-reported outcomes (PROs), defined as health outcomes directly reported by the patients who experienced them, and they provide vital information to both patients and doctors for use in the decision-making process. However, little information is available on other PROs, such as patient global assessment (PGA), sleep quality or work-related variables.

In this context, the aim of the systematic literature review reported here was to analyse the impact of comorbidity on PROs in PsA patients. We are confident that these results will help healthcare professionals in their management of this patient population.

\section{METHODS}

This article is based on previously conducted studies and does not contain any studies with human participants or animals performed by any of the authors.

A systematic review of the literature was conducted using a standardized, thorough and transparent approach, following the Cochrane dual-reviewer methodology [15] and PRISMA 
statement [16]. As the first step, an investigation protocol was established in which a group of experts in PsA collaborated in defining the research question, the PICO framework and the data to be collected. This group of experts supervised all parts of the systematic review of the literature and resolved any disagreements and uncertainties.

\section{Search Strategy}

As search strategy, two expert librarians performed a comprehensive and sensitive electronic search strategy of databases, including the MEDLINE, Embase, and the Cochrane database, in March 2019. MeSH (Medical Subject Headings) terms and free text terms were used. Only articles on human studies and those written in English and Spanish were searched. The search method is described in detail in the Electronic Supplementary Material.

The abstracts of the annual scientific meetings of the American College of Rheumatology (ACR) and The European League Against Rheumatism (EULAR) of 2017 and 2018 were also examined. All retrieved references were managed in Endnote (Clarivate Analytics, Philadelphia, PA, USA).

The last step in the search strategy was to conduct a hand search of the references of the included studies. Additional publications or other information provided by the experts related to the systematic review were also evaluated.

\section{Selection Criteria}

The studies retrieved using the above-mentioned strategies were included in our systematic review if they met a number of pre-specified criteria. First, the patients studied had to be PsA patients (according to international or medical criteria) and aged $\geq 18$ years; no restrictions were applied regarding disease duration, activity, severity or treatments. Second, PsA subjects with a comorbidity were included (irrespective of the type or number). Third, studies reporting PROs, including pain, function, PGA, quality of life, work disability or sleep disturbance, were included (irrespective of the type of measures). Only systematic literature reviews, randomized controlled trials and observational studies were included; basic science and animal studies were excluded.

\section{Screening of Studies and Data Collection}

Two reviewers independently screened the titles and abstracts of the retrieved articles to assess whether the selection criteria were satisfied. This process was performed in 20-min sessions. In case of discrepancy/disagreement over the eligibility of an article, a third reviewer was asked to resolve the disagreement. The same two reviewers also collected the data from the included studies using ad hoc standard forms. All data collected from each article were checked by both reviewers independently and then independently reviewed. If during this process the reviewers found any discrepancy, then a consensus was reached by looking at the original article or by asking the third reviewer. Articles that did not fulfil all of the inclusion criteria or that had insufficient data were excluded. A modification of the Oxford Centre for Evidence-Based Medicine (CEBM) levels of evidence, 2011 update [17], was used to grade the quality of the article. Evidence tables were then produced.

\section{Data Analysis and Presentation}

The main features of the studies included in the present systematic literature review are described in the evidence tables (see Results section). Depending on the variable, the results are expressed as a number and a percentage, mean and standard deviation or mean and interquartile range (p25-p75), and as odds ratio (OR), relative risk or hazard ratio, with $95 \%$ confidence intervals (CIs).

Meta-analysis was only planned when sufficient homogeneity was present among the included studies.

\section{RESULTS}

The search strategies retrieved 1155 articles, of which 82 were reviewed in detail in addition to 


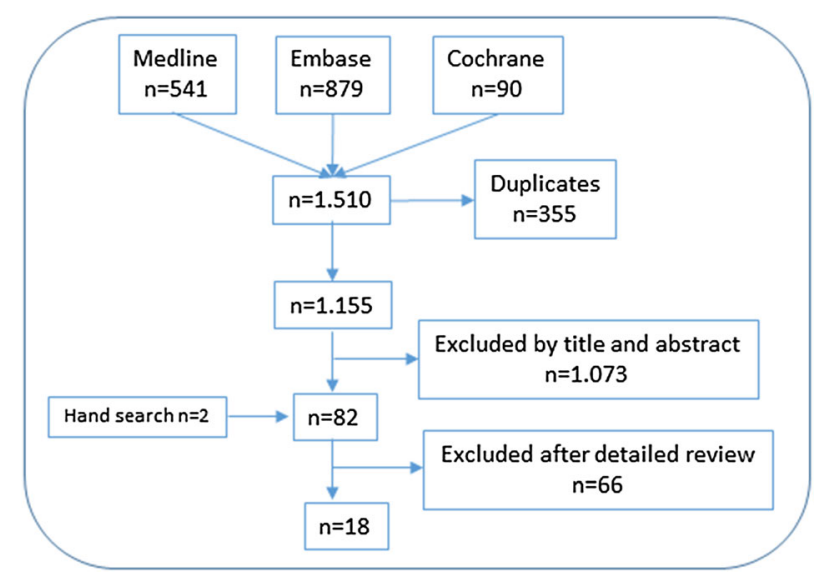

Fig. 1 Flowchart of study inclusion strategy

two additional studies identified from the hand search (Fig. 1). Ultimately, 66 of the 84 studies were excluded from inclusion in the systematic literature review (Table 1) [12, 18-82], and 18 articles were included $[9,14,83-98]$, one of which had been identified from the hand search (see Tables 2 and 3 for more detail).

Most of the studies included in the systematic literature review were cross-sectional $[9,84,85,87-90,92-97]$, although prospective observational studies were also found $[14,83,86,91,98]$, and most were of moderate quality (predominantly Oxford CEBM levels of evidence $2 \mathrm{~b}$ or 4 ). The total number of PsA patients included in each study ranged from 3571 [91] to 40 [97]. The majority of patients were middle-aged women, and almost all diagnoses were based onthe Classification Criteria for Psoriatic Arthritis (CASPAR) criteria. There was a great variability in the number and types of comorbidities. Some studies analysed the impact of an individual comorbidity, such as obesity [86, 98], FM $[9,94,96]$ or anxiety and depression [85, 87], but others analysed the impact of the number of comorbidities $[14,88,95]$, using indexes such as the Charlson Comorbidity Index (CCI).

The most frequently analysed PROs were function $[83,84,86,89,92,94]$, quality of life $[9,14,84,91-93]$ and fatigue [83, 85, 87, 93], but some studies evaluated the impact of comorbidity on pain $[83,84,91,98]$, work disability [88] or sleep quality [93, 96, 97]. Most of PROs were evaluated with validated questionnaires, such as the Health Assessment Questionnaire (HAQ) or the Bath Ankylosing Spondylitis Functional Index (BASFI) for the assessment of function or the 36 Item Short Form Health Survey (SF-36), the EuroQol 5 dimensions (EQ-5D) or Ankylosing Spondylitis Quality of Life Index (ASQoL) for quality of life. However, some studies on other PROs, such as pain or PGA, were analysed with a visual analogue scale or numerical scale.

The impact of comorbidity on PROs of PsA patients was examined using the CCI in two large, high-quality prospective cohorts $[83,86]$. In the first study (using DANBIO, a nationwide registry of biological therapies in Denmark), the authors noted that a higher score on the CCI was associated with worse function, pain, fatigue and PGA (univariate analyses) [83]. The second study, i.e. the CARMA study, was designed to evaluate cardiovascular risk in patients with chronic inflammatory rheumatic diseases [86]. In this study multivariate analyses were performed, and a higher CCI was also found to be associated with worse function, as assessed with HAQ (coefficient $\beta$ 0.09, $p=0.040$ ).

Three other studies used a list of comorbidities to evaluate the impact of the number of reported comorbidities on PROs in PsA patients $[14,88,95]$. One of these studies, a prospective observational study with more than 600 patients with PsA, found that the presence of at least three comorbidities significantly worsened a patient's quality of life, as assessed with the SF- 
Table 1 Studies excluded from systematic literature review and reasons for exclusion

\begin{tabular}{|c|c|c|}
\hline $\begin{array}{l}\text { Study } \\
\text { number } \\
\text { (arbitrary) }\end{array}$ & $\begin{array}{l}\text { Study (first author/ } \\
\text { year) }\end{array}$ & Reason for exclusion \\
\hline 1 & Abou-Raya/2014 [18] & No data regarding the impact of comorbidity on PROs \\
\hline 2 & Aguiar/2013 [20] & No data regarding the impact of comorbidity on PROs \\
\hline 3 & Aguiar/2013 [19] & No data regarding the impact of comorbidity on PROs \\
\hline 4 & Akhtari/2018 [21] & No data regarding the impact of comorbidity on PROs \\
\hline 5 & Aljohani/2017 [22] & No data regarding the impact of comorbidity on PROs \\
\hline 6 & Arancibia/2014 [24] & No data regarding the impact of comorbidity on PROs \\
\hline 7 & Arancibia/2015 [23] & No data regarding the impact of comorbidity on PROs \\
\hline 8 & Bandinelli/2009 [25] & No numerical data provided \\
\hline 9 & Batkaeva/2018 [26] & No data regarding the impact of comorbidity on PROs \\
\hline 10 & Bessette/2018 [27] & Comorbidities were eventually not analysed \\
\hline 11 & Bird/2017 [28] & Comorbidities were eventually not analysed \\
\hline 12 & Birra/2018 [29] & Obesity was not analysed regarding to any PROs \\
\hline 13 & Cano-Garcia/2016 [31] & No numerical/statistical data provided \\
\hline 14 & Cano-Garcia/2017 [30] & No numerical/statistical data provided \\
\hline 15 & Cano-Garcia/2018 [32] & No numerical/statistical data provided \\
\hline 16 & Carneiro/2010 [33] & $\begin{array}{l}\text { Congress abstract. The authors subsequently published an article which is } \\
\text { included in this systematic literature review }\end{array}$ \\
\hline 17 & Castañeda/2015 [34] & No data regarding the impact of comorbidity on PROs \\
\hline 18 & Cauli/2011 [35] & No data regarding the impact of comorbidity on PROs \\
\hline 19 & Cetin/2015 [36] & No numerical data provided \\
\hline 20 & Chimenti/2017 [38] & No data regarding the impact of comorbidity on PROs \\
\hline 21 & Chimenti/2019 [37] & No sub-analysis for PsA patients \\
\hline 22 & Da Cruz Ribeiro/2018 [39] & No numerical data provided \\
\hline 23 & $\operatorname{Dan} / 2011[40]$ & No sub-analysis for PsA patients \\
\hline 24 & De Gaspari/2015 [41] & No data regarding the impact of comorbidity on PROs \\
\hline 25 & Duffin/2009 [42] & No data regarding the impact of comorbidity on PROs \\
\hline 26 & Duruöz/2013 [44] & No data regarding the impact of comorbidity on PROs \\
\hline 27 & Duruoz/2018 [43] & No data regarding the impact of comorbidity on PROs \\
\hline 28 & Eder/2015 [45] & No data regarding the impact of comorbidity on PROs \\
\hline
\end{tabular}


Table 1 continued

\begin{tabular}{|c|c|c|}
\hline $\begin{array}{l}\text { Study } \\
\text { number } \\
\text { (arbitrary) }\end{array}$ & $\begin{array}{l}\text { Study (first author/ } \\
\text { year) }\end{array}$ & Reason for exclusion \\
\hline 29 & Farkas/2017_1 [46] & No data regarding the impact of comorbidity on PROs \\
\hline 30 & Farkas/2017_2 [47] & No data regarding the impact of comorbidity on PROs \\
\hline 31 & Fonti/2018 [48] & No sub-analysis for PsA patients \\
\hline 32 & Frede/2018 [49] & No data regarding the impact of comorbidity on PROs \\
\hline 33 & Gezer/2014 [50] & No numerical data provided \\
\hline 34 & Gossec/2011 [51] & No data regarding the impact of comorbidity on PROs \\
\hline 35 & Gottlieb/2019 [52] & PsA diagnosis notvalidated \\
\hline 36 & Grubišić/2013 [53] & No data regarding the impact of comorbidity on PROs \\
\hline 37 & Gulati/2018 [54] & No numerical/statistical data provided \\
\hline 38 & Haglund/2013 [55] & No sub-analysis for PsA patients \\
\hline 39 & Haroon/2013 [56] & No numerical data provided \\
\hline 40 & Huscher/2016 [57] & No data regarding the impact of comorbidity on PROs \\
\hline 41 & Husted/2010 [58] & No numerical/statistical data provided \\
\hline 42 & Husted/2012 [59] & No data regarding the impact of comorbidity on PROs \\
\hline 43 & Hyphantis/2013 [60] & No numerical/statistical data provided \\
\hline 44 & Khraishi/2014 [61] & No data regarding the impact of comorbidity on PROs \\
\hline 45 & Kotsis/2012 [62] & No data regarding the impact of comorbidity on PROs \\
\hline 46 & Kurizky/2012 [63] & No data regarding the impact of comorbidity on PROs \\
\hline 47 & Kuru/2014 [64] & No data regarding the impact of comorbidity on PROs \\
\hline 48 & Madeira/2017 [65] & No sub-analysis for PsA patients \\
\hline 49 & McDonough/2012 [66] & No data regarding the impact of comorbidity on PROs \\
\hline 50 & Meesters/2018 [67] & No data regarding the impact of comorbidity on PROs \\
\hline 51 & Michelsen/2017 [68] & No numerical/statistical data provided \\
\hline 52 & Miller/2013 [12] & No numerical/statistical data provided \\
\hline 53 & Nemes/2013 [69] & No data regarding the impact of comorbidity on PROs \\
\hline 54 & Ogdie/2014 [70] & Study design \\
\hline 55 & Pistone/2013 [71] & No data regarding the impact of comorbidity on PROs \\
\hline 56 & Rhee/2011 [74] & No numerical/statistical data provided \\
\hline 57 & Rhee/2012 [72] & Provides the same data as Rhee/2011 [56] \\
\hline
\end{tabular}


Table 1 continued

\begin{tabular}{lll}
\hline $\begin{array}{l}\text { Study } \\
\text { number } \\
\text { (arbitrary) }\end{array}$ & $\begin{array}{l}\text { Study (first author/ } \\
\text { year) }\end{array}$ & Reason for exclusion \\
\hline 58 & Rhee/2012_2 [73] & No data regarding the impact of comorbidity on PROs \\
59 & Rosen/2012 [75] & No data regarding the impact of comorbidity on PROs \\
60 & Salaff/2009 [76] & No data regarding the impact of comorbidity on PROs \\
61 & Sinnathurai/2018 [77] & No data regarding the impact of comorbidity on PROs \\
62 & Sinnathurai/2018 [78] & No numerical data provided \\
63 & Szentpetery/2016 [79] & No data regarding the impact of comorbidity on PROs \\
64 & Tillet/2013 [80] & No data regarding the impact of comorbidity on PROs \\
65 & Urruticoechea Arana/2015 & No numerical/statistical data provided \\
66 & {$[81]$} & No numerical/statistical data provided \\
\hline
\end{tabular}

Ps $A$ Psoriatic arthritis, PROs Patient-reported outcomes

36 (multivariate analyses), in both the physical and mental dimensions ( $\beta$ coefficients of -4.91 $[p<0.001]$ and $-2.84[p=0.005]$, respectively) [14]. A second study, a small prospective study, used the Functional Comorbidity Index (FCI) to corroborate that an increase in the number of comorbidities was significantly associated with moderate-severe work disability (OR 2.31, 95\% CI 1.19-4.50). The third study was a cross-sectional study that included 189 PsA patients [95]. The authors compared PsA patients with $\geq 5$ comorbidities to those with none and found that more comorbidities was associated with a worse BASFI score ( $\beta$ coefficient 2.33, $p=0.040$ ).

The remaining studies included in the systematic literature review analysed the impact of individual comorbidities on patient PROs. In three such studies, the authors focussed on the impact of FM on patient PROs $[9,94,96]$. In the small prospective study of Brikman et al. [9], the presence of FM (vs. its absence) was associated with worse median HAQ scores (1.75 vs. 0.25 , $p<0.001)$ and worse median BASDAI $(7.18$ vs. $2.87, p<0.001)$. We also included a cross-sectional study that in addition to evaluating the presence of FM also evaluated the impact of the extent and severity of the disease using the Widespread Pain Index (WPI) and the Symptom Severity Scale (SSS) [94]. Similar to the results reported by Brikman et al. [9], patients with FM had significantly worse scores on the HAQ (unadjusted data). In addition, the WPI was strongly correlated with the BASDAI ( $r=0.804$, $p<0.001)$, although the correlation with the HAQ was weak $(r=0.478, p<0.001)$. The SSS score also showed a weak correlation with the HAQ $(r=0.3, p<0.001)$. Another small crosssectional study that included $50 \mathrm{FM}$ patients used the Fibromyalgia Impact Questionnaire (FIQ) to assess the impact of FM on patient PROs [96]. The authors of this study reported a moderate correlation between the FIQ and the Pittsburgh Sleep Quality Index (PSQI) $(r=0.56$, $p<0.001$ ) and a moderate-strong correlation with quality of life (as measured on the Psoriatic Arthritis Quality of Life questionnaire [PsAQoL]) $(r=0.69, p<0.001)$.

Anxiety and depression were examined in two small cross-sectional studies using the Hospital Anxiety and Depression Scale (HADS) questionnaire [85, 87]. Both studies found 
Table 2 Evidence table constructed, showing main characteristics of studies included in the systematic literature review

\begin{tabular}{|c|c|c|c|c|c|}
\hline $\begin{array}{l}\text { Study } \\
\text { number of } \\
\text { included } \\
\text { study } \\
\text { (arbitrary) }\end{array}$ & $\begin{array}{l}\text { Study (first author/ } \\
\text { year) and type of } \\
\text { study }\end{array}$ & Population & Comorbidity & PROs & Quality $^{a}$ \\
\hline 1 & $\begin{array}{l}\text { Ballegaard/2018 [83], } \\
\text { observational } \\
\text { prospective cohort } \\
\text { (DANBIO } \\
\text { registry) }\end{array}$ & $\begin{array}{l}n=1750 \text { PsA patients; } 55 \% \\
\text { women; mean age } 48 \text { years; PsA } \\
\text { duration } 1-11 \text { years; CCI } 0 \\
(61 \%), 1(28 \%), \geq 2(11 \%)\end{array}$ & $\mathrm{CCI}$ & $\begin{array}{l}\text { Function } \\
\text { (HAQ) } \\
\text { PGA (VAS } \\
0-100) \\
\text { Pain (VAS } \\
0-100) \\
\text { Fatigue (VAS } \\
0-100)\end{array}$ & $2 \mathrm{~b}$ \\
\hline 2 & $\begin{array}{l}\text { Bremander/2014 } \\
\text { [84], cross- } \\
\text { sectional study }\end{array}$ & $\begin{array}{l}n=1.73 \text { PsA patients; } 58 \% \\
\text { women; mean age } 57 \text { years; } \\
\text { duration PsA } 12 \text { years; } 38 \% \\
\text { never smoking, } 62 \% \text { yes (18\% } \\
\text { current, } 44 \% \text { ex-smokers) }\end{array}$ & $\begin{array}{l}\text { Smoking (self } \\
\text { declared) }\end{array}$ & $\begin{array}{l}\text { Quality of } \\
\text { life (EQ- } \\
\text { 5D) } \\
\text { Function } \\
\text { (HAQ) } \\
\text { Global health } \\
\text { (numeric } \\
\text { scale 0-10) } \\
\text { Fatigue } \\
\text { (numeric } \\
\text { scale 0-10) } \\
\text { Pain } \\
\text { (numeric } \\
\text { scale } 0-10 \text { ) }\end{array}$ & 4 \\
\hline 3 & $\begin{array}{l}\text { Brikman/2016 [9], } \\
\text { cross-sectional } \\
\text { study }\end{array}$ & $\begin{array}{l}n=73 \text { PsA patients (CASPAR } \\
\text { criteria; } 57 \% \text { women; mean age } \\
52 \text { years; PsA duration } 10 \text { years; } \\
17.8 \% \text { FM }\end{array}$ & $\begin{array}{l}\text { FM (ACR } 2010 \text { and } \\
1990 \text { criteria) }\end{array}$ & $\begin{array}{l}\text { Function } \\
\text { (HAQ) } \\
\text { Quality of } \\
\text { life (DLQI) }\end{array}$ & $3 a$ \\
\hline 4 & $\begin{array}{l}\text { Carneiro/2017 [85], } \\
\text { cross-sectional } \\
\text { study }\end{array}$ & $\begin{array}{c}n=101 \text { PsA patients (CASPAR } \\
\text { criteria); } 44 \% \text { women; mean age } \\
51 \text { years; mean HADS } 7.39\end{array}$ & $\begin{array}{l}\text { Anxiety and } \\
\text { depression } \\
\text { (HADS) }\end{array}$ & $\begin{array}{l}\text { Fatigue } \\
\text { (FACIT- } \\
\text { F) }\end{array}$ & 4 \\
\hline 5 & $\begin{array}{l}\text { Fernández- } \\
\text { Carballido/2018 } \\
\text { [86], observational } \\
\text { prospective cohort }\end{array}$ & $\begin{array}{l}n=721 \text { PsA patients; mean CCI } \\
1.30\end{array}$ & $\begin{array}{l}\text { CCI } \\
\text { Obesity (BMI) }\end{array}$ & $\begin{array}{l}\text { Function } \\
\text { (HAQ) }\end{array}$ & $2 \mathrm{a}$ \\
\hline
\end{tabular}


Table 2 continued

\begin{tabular}{|c|c|c|c|c|c|}
\hline $\begin{array}{l}\text { Study } \\
\text { number of } \\
\text { included } \\
\text { study } \\
\text { (arbitrary) }\end{array}$ & $\begin{array}{l}\text { Study (first author/ } \\
\text { year) and type of } \\
\text { study }\end{array}$ & Population & Comorbidity & PROs & Quality $^{a}$ \\
\hline 6 & $\begin{array}{l}\text { Gubar_2018 [87], } \\
\text { cross-sectional } \\
\text { study }\end{array}$ & $\begin{array}{l}n=78 \text { early PsA patients } \\
\text { (CASPAR criteria); } 50 \% \\
\text { women; mean age } 36 \text { years; } \\
\text { mean anxiety score } 5.7 ; \text { mean } \\
\text { depression score } 3.8\end{array}$ & $\begin{array}{l}\text { Anxiety and } \\
\text { depression } \\
\text { (HADS) }\end{array}$ & $\begin{array}{l}\text { Fatigue } \\
\quad \text { (FACIT- } \\
\text { F) } \\
\text { Function } \\
\text { (HAQ) }\end{array}$ & 4 \\
\hline 7 & $\begin{array}{l}\text { Husted/2013 [14], } \\
\text { cross-sectional } \\
\text { study }\end{array}$ & $\begin{array}{l}n=631 \text { PsA patients(CASPAR } \\
\text { criteria); } 41 \% \text { women; mean age } \\
50 \text { years; PsA duration } 13 \text { years; } \\
\text { mean number of comorbidities } \\
2.5\end{array}$ & $\begin{array}{l}\text { Presence of } 15 \\
\text { comorbidities }\end{array}$ & $\begin{array}{l}\text { Quality of } \\
\text { life (SF-36) }\end{array}$ & $3 a$ \\
\hline 8 & $\begin{array}{l}\text { Kennedy/2014 [88], } \\
\text { cross-sectional } \\
\text { study }\end{array}$ & $\begin{array}{l}n=146 \text { PsA patients (CASPAR } \\
\text { criteria); } 39 \% \text { women; mean age } \\
50 \text { years; PsA duration } 14 \text { years; } \\
\text { mean FCI } 1.08\end{array}$ & $\begin{array}{l}\text { FCI comorbidity } \\
\text { index }\end{array}$ & $\begin{array}{l}\text { Work } \\
\text { disability } \\
\text { (WLQ) }\end{array}$ & 4 \\
\hline 9 & $\begin{array}{l}\text { Oten/2017 [89], } \\
\text { cross-sectional } \\
\text { study }\end{array}$ & $\begin{array}{l}n=58 \text { PsA patients (CASPAR } \\
\text { criteria); } 57 \% \text { women; mean age } \\
42 \text { years; PsA duration } 16 \text { years; } \\
31 \% \text { osteoporosis }\end{array}$ & $\begin{array}{l}\text { Osteoporosis } \\
\text { (DEXA) }\end{array}$ & $\begin{array}{l}\text { BASDAI } \\
\text { Function } \\
(\mathrm{HAQ})\end{array}$ & 4 \\
\hline 10 & $\begin{array}{l}\text { Pehlevan/2014 [90], } \\
\text { cross-sectional } \\
\text { study }\end{array}$ & $\begin{array}{l}n=55 \text { PsA patients (CASPAR } \\
\text { criteria); } 62 \% \text { women; mean age } \\
46 \text { years; } 24 \% \text { metabolic } \\
\text { syndrome }\end{array}$ & $\begin{array}{l}\text { Metabolic syndrome } \\
\text { (NCEP-ACT III } \\
\text { criteria) }\end{array}$ & $\begin{array}{l}\text { BASDAI } \\
\text { Function } \\
\text { (HAQ, } \\
\text { RAPID-3) }\end{array}$ & 4 \\
\hline 11 & $\begin{array}{l}\text { Piche/2015 [91], } \\
\text { observational } \\
\text { prospective cohort }\end{array}$ & $\begin{array}{l}n=3.571 \text { PsA patients; } 36 \% \\
\text { women; mean age } 50 \text { years; PsA } \\
\text { duration } 14 \text { years }\end{array}$ & $\begin{array}{l}\text { Smoking (past, } \\
\text { current, duration) } \\
\text { Alcohol intake (past, } \\
\text { current, duration) }\end{array}$ & $\begin{array}{l}\text { Function } \\
\text { (HAQ, } \\
\text { FSS, } \\
\text { BASFI) } \\
\text { Pain (HAQ) } \\
\text { Fatigue } \\
\text { (FACIT) } \\
\text { Quality of } \\
\text { life } \\
\text { (EQ 5D, } \\
\text { DLQI, } \\
\text { ASQoL, } \\
\text { SF-36) }\end{array}$ & $2 \mathrm{~b}$ \\
\hline
\end{tabular}


Table 2 continued

\begin{tabular}{|c|c|c|c|c|c|}
\hline $\begin{array}{l}\text { Study } \\
\text { number of } \\
\text { included } \\
\text { study } \\
\text { (arbitrary) }\end{array}$ & $\begin{array}{l}\text { Study (first author/ } \\
\text { year) and type of } \\
\text { study }\end{array}$ & Population & Comorbidity & PROs & $\overline{\text { Quality }}^{\mathbf{a}}$ \\
\hline 12 & $\begin{array}{l}\text { Sanci/2018 [92], } \\
\text { cross-sectional } \\
\text { study }\end{array}$ & $\begin{array}{l}n=104 \text { PsA patients (CASPAR } \\
\text { criteria); } 63.5 \% \text { women; mean } \\
\text { age } 50 \text { years; PsA duration } \\
8 \text { years; } 45.2 \% \text { metabolic } \\
\text { syndrome }\end{array}$ & $\begin{array}{l}\text { Metabolic syndrome } \\
\text { (NCEP-ACT III } \\
\text { criteria) }\end{array}$ & $\begin{array}{l}\text { BASDAI } \\
\text { Function } \\
\text { (BASFI, } \\
\text { HAQ) } \\
\text { PGA (VAS } \\
0-100) \\
\text { Quality of } \\
\text { life (DLQI, } \\
\text { ASQoL) }\end{array}$ & 4 \\
\hline 13 & $\begin{array}{l}\text { Sandikci/2018 [93], } \\
\text { cross-sectional } \\
\text { study }\end{array}$ & $\begin{array}{l}n=50 \text { PsA patients (CASPAR } \\
\text { criteria); } 33 \% \text { women; mean age } \\
45 \text { years; } 64 \% \text { restless legs } \\
\text { syndrome }\end{array}$ & $\begin{array}{l}\text { Restless legs } \\
\text { syndrome (RLS } \\
\text { Study Group } \\
\text { recommendations) }\end{array}$ & $\begin{array}{l}\text { Fatigue (FSS) } \\
\text { Quality of } \\
\text { life (SF-36) } \\
\text { Sleep quality } \\
\text { (PSQI) }\end{array}$ & 4 \\
\hline 14 & $\begin{array}{l}\text { Sharma/2017 [94], } \\
\text { cross-sectional } \\
\text { study }\end{array}$ & $\begin{array}{l}n=102 \text { PsA patients; } 50 \% \\
\text { women; mean age } 43 \text { years }\end{array}$ & FM (WPI, SSS) & $\begin{array}{l}\text { BASDAI } \\
\text { Function } \\
\text { (PROMIS- } \\
\text { HAQ) }\end{array}$ & 4 \\
\hline 15 & $\begin{array}{l}\text { Tanner/2014 [95], } \\
\text { cross-sectional } \\
\text { study }\end{array}$ & $\begin{array}{l}n=189 \text { PsA patients; } 52 \% \\
\text { women; mean age } 50 \text { years; } \\
75 \% \text { comorbidity }\end{array}$ & $\begin{array}{l}\text { Number of } \\
\text { comorbidities }\end{array}$ & BASFI & 4 \\
\hline 16 & $\begin{array}{l}\text { Ulutatar/2018 [96], } \\
\text { cross-sectional } \\
\text { study }\end{array}$ & $\begin{array}{l}n=50 \text { PsA patients (CASPAR } \\
\text { criteria); 64\% women; mean age } \\
49 \text { years; PsA duratation } \\
90 \text { months; } 64 \% \text { FM }\end{array}$ & FM (FIQ) & $\begin{array}{l}\text { Quality of } \\
\text { life (PSQI) } \\
\text { Quality of } \\
\text { life } \\
\text { (PsASQoL) }\end{array}$ & 4 \\
\hline 17 & $\begin{array}{l}\text { Unal/2018 [97], } \\
\text { cross-sectional } \\
\text { study }\end{array}$ & $\begin{array}{l}n=40 \text { PsA, patients; } 68 \% \\
\text { women; mean age } 50 \text { years; PsA } \\
\text { duration } 99 \text { months; } 30 \% \\
\text { neuropathic pain }\end{array}$ & $\begin{array}{c}\text { Neuropathic pain } \\
\text { (painDETECT } \\
\text { questionnaire) }\end{array}$ & $\begin{array}{l}\text { Quality of } \\
\text { life (PSQI) } \\
\text { Quality of } \\
\text { life } \\
\text { (PsASQoL) }\end{array}$ & 4 \\
\hline
\end{tabular}


Table 2 continued

\begin{tabular}{|c|c|c|c|c|c|}
\hline $\begin{array}{l}\text { Study } \\
\text { number of } \\
\text { included } \\
\text { study } \\
\text { (arbitrary) }\end{array}$ & $\begin{array}{l}\text { Study (first author/ } \\
\text { year) and type of } \\
\text { study }\end{array}$ & Population & Comorbidity & PROs & Quality $^{a}$ \\
\hline 18 & $\begin{array}{l}\text { Zaffarana/2016 [98], } \\
\text { observational } \\
\text { prospective cohort } \\
\text { (RPSAODIA } \\
\text { registry) }\end{array}$ & $\begin{array}{l}n=110 \text { PsA patients (CASPAR } \\
\text { criteria); } 64 \% \text { women; mean age } \\
49 \text { years; PsA duratation } \\
90 \text { months; } 37.3 \% \text { obesity }\end{array}$ & Obesity (BMI) & $\begin{array}{l}\text { BASFI } \\
\text { Pain (VAS } \\
0-10)\end{array}$ & $3 a$ \\
\hline
\end{tabular}

ACR American College of Rheumatology, ASQoL Ankylosing Spondylitis Quality of Life Index, BASDAI Bath Ankylosing Spondylitis Disease Activity Index, BASFI Bath Ankylosing Spondylitis Functional Index, BMI body mass index, CASPAR Classification Criteria for Psoriatic Arthritis, $C C I$ Charlson Comorbidity Index, $D E X A$ dual-energy X-ray absorptiometry, $D L Q I$ Dermatology Quality of Life Index, EQ-5D EuroQol 5 dimensions, FACIT Functional Assessment of Chronic Illness Therapy scale, FACIT-F Functional Assessment of Chronic Illness Therapy-Fatigue scale, FCI Functional Comorbility Index, FIQ Fibromyalgia Impact Questionnaire, FM fibromyalgia, FSS Fatigue Severity Scale, HADS Hospital Anxiety and Depression Scale, HAQ Health Assessment Questionnaire, NCEP National Commission for Employment Policy, PGA patient global assessment, PROMIS Patient-Reported Outcomes Measurement Information System, PsASQol Psoriatic Arthritis Quality of Life questionnaire, PSQI Pittsburgh Sleep Quality Index, SF-36 36 Item Short Form Health Survey, SSS Symptom Severity Scale, VAS visual analogic scale, WPI Widespread Pain Index, WLQ Work Limitations Questionnaire ${ }^{a}$ Quality assessed with the 2011 Oxford Centre for Evidence-Based Medicine (CEBM) levels of evidence [17]

weak-moderate correlations with function and fatigue.

The impact of the metabolic syndrome on PROs of PsA patients, according to the National Commission for Employment Policy (NCEP)ACT III guidelines, was evaluated in two crosssectional studies, which recruited more than 150 patients with PsA [90, 92]. In one of these studies [90], no statistically significant association was found between the metabolic syndrome and the BASDAI, HAQ and Routine Assessment of Patient Index Data 3 (RAPID3) index, although it should be noted that the small size of the study sample (50 patients) may have influenced this result [90]. In the second study, the authors observed a negative impact of metabolic syndrome (compared with patients without it; higher values indicating more active disease/worse function/great disability) on the BASDAI (22 vs. $11, p=0.042$ ), BASFI (18 vs. 5 , $p=0.009)$, HAQ (0.375 vs. 0.125, $p=0.011)$,
PGA (31 vs. $20, p=0.030$ ), and ASQoL (7 vs. 3 , $p=0.017$ ) [92].

The impact of obesity on patients with PsA was analysed in two prospective cohorts included in this systematic literature review. One was the CARMA study (described earlier in this review; [86]) and the other was the PsA registry of Argentina (RAPSODIA registry [98]). The multivariate analyses of the CARMA cohort revealed that obesity was significantly associated with worse patient function (HAQ; $\beta$ coefficient $0.09, p=0.005$ ) [86], while the bivariate analyses of the RAPSODIA registry also found a significant negative association with function (BASFI) and pain [98].

The effect of smoking on PsA patient PROs was examined in two studies [84, 91]. One of these studies was a cross-sectional study that included more than 1000 patients with PsA [84]. The multivariate analyses comparing smokers and ex-smokers with patients who have never smoked demonstrated that smoking was 
Table 3 Main results of the studies included in the systematic literature review

\begin{tabular}{|c|c|c|}
\hline $\begin{array}{l}\text { Number of study } \\
\text { (arbitrary) }\end{array}$ & $\begin{array}{l}\text { Study (first author/ } \\
\text { year) }\end{array}$ & Results \\
\hline 1 & $\begin{array}{l}\text { Ballegaard/2018 } \\
\quad[83]\end{array}$ & $\begin{array}{l}\text { CCI } 0 \text { vs. } 1 \text { vs. } \geq 2 \\
\text { HAQ } 0.88 \text { vs. } 1.1 \text { vs. } 1.4(p<0.001) \\
\text { PGA } 68 \text { vs. } 69 \text { vs. } 75(p=0.021) \\
\text { Pain } 60 \text { vs. } 62 \text { vs. } 69(p=0.008) \\
\text { Fatigue } 65 \text { vs. } 67 \text { vs. } 78(p=0.002)\end{array}$ \\
\hline 2 & $\begin{array}{l}\text { Bremander/2014 } \\
\quad[84]\end{array}$ & $\begin{array}{l}\text { Current smokers/ex-smokers vs. never smoking (multivariate analyses) } \\
\text { EQ-5D } \beta \text { coefficient }-0.04(p=0.009) \\
\text { HAQ } \beta \text { coefficient } 0.043(p=0.210) \\
\text { Global Health } \beta \text { coefficient } 0.36(p=0.010) \\
\text { Pain } \beta \text { coefficient } 0.38(p=0.010) \\
\text { Fatigue } \beta \text { coefficient } 0.34(p=0.040) \\
\text { Pain locations } \beta \text { coefficient } 0.54(p=0.04) \\
\text { Current smokers vs. ex-smokers worse EQ-5D }(p=0.02) \text { and number of pain } \\
\text { locations }(p=0.04)\end{array}$ \\
\hline 3 & Brikman/2016 [9] & $\begin{array}{l}\text { FM vs. no FM } \\
\text { HAQ median (IQR) } 1.75(1.07-237) \text { vs. } 0.25(0-1) . p<0.00 \\
\text { BASDAI (mean } \pm \text { SD) } 7.18 \pm 1.73 \text { vs. } 2.87 \pm 2.35(p<0.001)\end{array}$ \\
\hline 4 & Carneiro/2017 [85] & $\begin{array}{l}\text { Correlation HADS and FACIT-F } \\
\text { HADS (anxiety) } r=-0.306(p=0.002) \\
\text { HADS (depression) } r=-0.339(p<0.001)\end{array}$ \\
\hline 5 & $\begin{array}{l}\text { Fernández- } \\
\text { Carballido/2018 } \\
{[86]}\end{array}$ & $\begin{array}{l}\text { Obesity (multivariate analyses) } \\
\text { HAQ } \beta \text { coefficient } 0.09(p=0.005) \\
\text { Higher CCI (multivariate analyses) } \\
\text { HAQ } \beta \text { coefficient } 0.09(p=0.040)\end{array}$ \\
\hline 6 & Gubar/2018 [87] & $\begin{array}{l}\text { Correlation HADS and HAQ } \\
\text { HADS (anxiety) } r=0.26 \\
\text { HADS (depression) } r=0.33 \\
\text { Correlation HADS and FACIT-F } \\
\text { HADS (anxiety) } r=-0.49 \\
\text { HADS (depression) } r=-0.48\end{array}$ \\
\hline
\end{tabular}


Table 3 continued

\begin{tabular}{|c|c|c|}
\hline $\begin{array}{l}\text { Number of study } \\
\text { (arbitrary) }\end{array}$ & $\begin{array}{l}\text { Study (first author/ } \\
\text { year) }\end{array}$ & Results \\
\hline 7 & Husted/2013 [14] & $\begin{array}{l}\text { Presence } 3 \text { comorbidities (multivariate analyses) } \\
\text { SF-36 (physical component) } \beta \text { coefficient }-4.91(p<0.001) \\
\text { SF-36 (mental component) } \beta \text { coefficient }-2.84(p=0.005)\end{array}$ \\
\hline 8 & Kennedy/2014 [88] & $\begin{array}{l}\text { Correlation FCI and WLQ } r=0.30(p<0.001) \\
\text { Moderate-severr vs. mild-none work disability (multivariate analyses) } \\
\text { FCI ( } 1 \text { unit increment) OR }=2.31(95 \% \text { CI } 1.19-4.50)\end{array}$ \\
\hline 9 & Öten/2017 [89] & $\begin{array}{l}\text { Osteoporosis vs. no osteoporosis } \\
\text { BASDAI (mean } \pm \text { SD) } 5.08 \pm 2.53 \text { vs. } 4.08 \pm 2.34(p=n s) \\
\text { HAQ (mean } \pm \text { SD) } 0.64 \pm 0.63 \text { vs. } 0.44 \pm 0.44(p=n s)\end{array}$ \\
\hline 10 & Pehlevan/2014 [90] & $\begin{array}{l}\text { Metabolic syndrome vs. no metabolic syndrome } \\
\text { BASDAI (mean } \pm \text { SD) } 3.9 \pm 2.1 \text { vs. } 3.8 \pm 2.4(p=\mathrm{ns}) \\
\text { HAQ (mean } \pm \text { SD) } 2.2 \pm 2 \text { vs. } 1.6 \pm 1.5(p=\mathrm{ns}) \\
\text { RAPID } 3 \text { (mean } \pm \text { SD) } 5.8 \pm 1.2 \text { vs. } 6.8 \pm 1.1(p=\mathrm{ns})\end{array}$ \\
\hline 11 & Piche/2015 [91] & $\begin{array}{l}\text { Correlation smoking (past, current, duration) } \\
\text { HAQ, FSS, BASFI, pain, FACIT, BASDAI, ASQoL, SF-36 (physical and } \\
\text { mental component) } r^{2}<0.2(p<0.050) \\
\text { DLQI }(p=\mathrm{ns}) \\
\text { Correlation current alcohol intake } \\
\text { HAQ, FSS, BASFI, pain, FACIT, BASDAI, ASQoL, EQ 5D, DLQI, SF-36 } \\
\text { (physical and mental component) } r^{2}<0.2(p<0.050) \\
\text { Alcohol intake at PsA diagnosis } \\
\text { SF-36 (physical component) } r^{2}<0.1(p<0.050)\end{array}$ \\
\hline 12 & Sanci/2018 [92] & $\begin{array}{l}\text { Metabolic syndrome vs. no metabolic syndrome } \\
\text { BASDAI median } 22 \text { vs. } 11(p=0.042) \\
\text { BASFI median } 18 \text { vs. } 5(p=0.009) \\
\text { HAQ median } 0.375 \text { vs. } 0.125(p=0.011) \\
\text { PGA median } 31 \text { vs. } 20(p=0.030) \\
\text { DLQI median } 0 \text { vs. } 1(p=\text { ns) } \\
\text { ASQoL median } 7 \text { vs. } 3(p=0.017)\end{array}$ \\
\hline
\end{tabular}


Table 3 continued

Number of study Study (first author/ Results
(arbitrary) year)

FSS $r 0.324(p=0.001)$

PSQI $r 0.305(p=0.001)$

SF-36 (physical component) $r=-0.418(p=0.001)$

SF-36 (mental component) $r=-0.212(p=0.010)$

Restless legs syndrome vs. no syndrome (multivariate analyses)

FSS $\beta$ coefficient $0.243(p=0.003)$

PSQI $\beta$ coefficient $0.269(p=0.002)$

SF-36 (physical component) $\beta$ coefficient $0.242(p=0.004)$

PROMIS HAQ (mean \pm SD) $49.26 \pm 18.95$ vs. $28.88 \pm 13.42(p<0.001)$

HAQ-Pain (mean \pm SD) $64.74 \pm 11.72$ vs. $43.37 \pm 13.36(p<0.001)$

HAQ-Health (mean \pm SD) $52.11 \pm 14.74$ vs. $24.10 \pm 14.52(p<0.001)$

Correlation WPI

BASDAI $r=0.804(p<0.001)$

PROMIS HAQ $r=0.478(p<0.001)$

Correlation SSS

PROMIS HAQ $r=0.3(p<0.001)$

BASFI according to the number of comorbidities

$\geq 5$ comorbidities vs. $0 \beta$ coefficient $2.33(p=0.040)$

$\geq 5$ comorbidities vs. $1 \beta$ coefficient 2.51 ( $p=\mathrm{ns}$ )

$\geq 5$ comorbidities vs. $2 \beta$ coefficient $2.18(p=0.030)$

$\geq 5$ comorbidities vs. $3 \beta$ coefficient 3.84 ( $p=\mathrm{ns}$ )

$\geq 5$ comorbidities vs. $4 \beta$ coefficient 4.20 ( $p=n$ s)

16

Ulutatar/2018 [96] Correlation FIQ

PSQI $r=0.56(p<0.001)$

PsAQoL $r=0.69(p<0.001)$

17

Unal/2018 [97] Correlation painDETECT

PSQI $r=0.40(p=0.010)$

PsAQoL $r=0.66(p=0.001)$ 
Table 3 continued

\begin{tabular}{lll}
\hline $\begin{array}{l}\text { Number of study } \\
\text { (arbitrary) }\end{array}$ & $\begin{array}{l}\text { Study (first author/ } \\
\text { year) }\end{array}$ & Results \\
\hline 18 & Zaffarana/2016 [98] & Obesity vs. normal weight \\
& & BASFI (mean \pm SD) $54.4 \pm 2.8$ vs. $2.7 \pm 2.5(p=0.030)$ \\
& Pain (mean \pm SD) $6.7 \pm 7.6$ vs. $4.6 \pm 2.4(p=0.050)$ \\
\hline
\end{tabular}

CI Confidence interval, ns non-significant, OR odds ratio, RAPID Routine Assessment of Patient Index Data 3 index

significantly associated with the EQ-5D $(\beta$ coefficient $-0.04, p=0.009$ ), the HAQ ( $\beta$ coefficient $0.043, p=0.210$ ), overall health ( $\beta$ coefficient $0.36, p=0.010)$, pain ( $\beta$ coefficient 0.38 , $p=0.010)$, fatigue ( $\beta$ coefficient $0.34, p=0.040$ ) and pain location ( $\beta$ coefficient $0.54, p=0.04$ ) [84]. The second study was a prospective cohort of 3571 PsA patients [91]. The results showed a weak correlation between smoking (past and current) and HAQ, Fatigue Severity Scale (FSS), BASFI, pain, Functional Assessment of Chronic Illness Therapy (FACIT), BASDAI, ASQoL and SF-36 (physical and mental component) $\left(r^{2}<0.2, p<0.050\right)$ [77]. This study also evaluated current alcohol consumption and found that it was also weakly correlated with $\mathrm{HAQ}$, FSS, BASFI, pain, FACIT, BASDAI, ASQoL, EQ$5 \mathrm{D}$, Dermatology Quality of Life Index (DLQI), and SF-36 (physical and mental component) $\left(r^{2}<0.2, p<0.050\right)$ [91].

This systematic literature review identified a small cross-sectional study that examined the impact of osteoporosis (detected using dualenergy X-ray absorptiometry [DEXA]) on 58 patients with PsA [89]. A comparison of PsA patients with and without this comorbidity did not detect any statistically significant differences in the BASDAI $(5.08 \pm 2.53$ vs. $4.08 \pm 2.34, p>0.050)$ and HAQ scores $(0.64 \pm 0.63$ vs. $0.44 \pm 0.44, p>0.050)$. However, the small size of the study sample may have influenced these results.

A study was also selected for inclusion in our systematic literature review which enrolled 50 PsA patients with restless legs syndrome [93]. In the multivariate analyses and in the comparison with patients who did not have the syndrome, an association was found with the FSS ( $\beta$ coefficient $0.243, p=0.003$ ), PSQI ( $\beta$ coefficient $0.299, p=0.002$ ) and the SF-36 (physical component) ( $\beta$ coefficient $0.242, p=0.004)$.

Finally, a cross-sectional study with PsA patients diagnosed with neuropathic pain was also included in this review. These patients were evaluated using the painDETECT questionnaire [97]. The authors reported that neuropathic pain was moderately correlated with the PSQI $(r=0.40, p=0.010)$ and with the PsAQoL $(r=0.66, p<0.001)$.

\section{DISCUSSION}

Our evaluation of the studies compiled in our systematic literature review confirms that comorbidity has a great impact on PROs in PsA patients.

It should be highlighted that, compared with the number of prevalence studies published on comorbidity and PsA or of those analysing the impact on disease activity or treatment, the number of studies that examine the impact of comorbidity on patient PROs is significantly lower. It is also important to note that much of the current evidence comes from abstracts of scientific meetings that are not subsequently published in the main bibliographic databases. Moreover, the impact of comorbidity on PROs is usually a sub-analysis and not the main objective of the study. Taking also into account the increasing size of the aging population and improvements in the recognition of comorbidity and its treatments, it is clear that more research is needed to properly estimate the real impact of comorbidity on PsA. 
We included 18 articles in our systematic literature review, but many of these described small observational studies of moderate quality, some of which were reported in scientific meetings $[89,94]$. However, the results of these studies are in line with those depicted in the cohort studies $[83,86]$, reinforcing the validity of their findings.

Our evaluation of the selected studies revealed a great variability in the number and type of comorbidities studied, as well as in their definitions and measures. In some studies the impact of an individual comorbidity was examined, such as obesity $[86,98]$, FM $[9,96]$ or anxiety and depression [85, 87], while in other studies the impact of the number of comorbidities was examined [14, 95], using indexes such as the CCI or FCI. With respect to the PROs, we included a very long list in our review, including function $[83,86,92]$, quality of life $[14,84,93]$, fatigue $[83,85]$, pain $[83,91,98]$, work disability [88] and/or or sleep quality [93, 6, 97].

We also considered in our evaluation the impact of comorbidity on PROs as observed both in early (though preliminary data) and established PsA. At the time of disease diagnosis, comorbidity might not only be present but also have a relevant impact on PROs, possibly having important consequences in the clinical setting in terms of identifying the comorbidities and treating them as soon as possible.

As expected, we found that the impact of comordidity was greater with an increasing number of comorbidities, as well as with the severity of the comorbidity $[83,86]$. Our analysis showed that even just one comorbidity had an impact on the PROs, but we were unable to determine which comorbidity had the greatest impact. We found good-quality evidence showing that obesity might have a negative effect on relevant PROs like function and pain. The same level of evidence examined the effect of smoking (smokers and ex-smokers) on different PROs, including pain, function, fatigue, quality of life and overall health. The multivariate analyses described a significant impact of comorbidity on all of these aspects. Data from low-quality studies suggested that metabolic syndrome might also have a negative impact on BASDAI, function, PGA and quality of life.

Some studies used correlations to assess the impact of comorbidity on PROs in PsA patients. The results from these studies were variable. For example, anxiety and depression wer found to have weak-moderate correlations with function and fatigue. The correlations between FM and BASDAI, function, quality of sleep and quality of life were weak, moderate or strong depending on the PROs. Also, although the impact of alcohol intake on PROs in PsA patients was statistically significant, its correlations with pain, function, fatigue, quality of life and overall health were weak.

Finally, the impact of other comorbidities, such as osteoporosis, restless legs syndrome and neuropathic pain, was also described in the studies included in our systematic literature review, but more data are required to establish their impact on PROs in PsA patients.

There are a number of limitations to the present systematic literature review, of which the most important is the inter-study variability regarding the definition of comorbidities and PROs and the associated criteria and measures; this variability limits the comparability of results among studies. A second limitation is the lack of studies specifically designed to assess the impact of comorbidity on the PROs in PsA patients; such analyses are mostly secondary analyses in the studies included in our systematic literature review.

\section{CONCLUSIONS}

In summary, there is sufficient evidence demonstrating the high prevalence of comorbidity $[2,4,5]$ in patients with PsA and its impact on outcome measures, treatment response and clinical decision-making [6, 9-13]. A number of studies have also highlighted that comorbidity clearly impairs function and quality of life in PsA patients. Our systematic literature review demonstrates the impact of comorbidity on a wide variety of PROs, such as work disability or sleep quality. We conclude that recognizing and addressing comorbidities 
is critical to providing safe and effective treatment to patients with PsA.

\section{ACKNOWLEDGEMENTS}

Funding. Pfizer S.L.U. has unconditionally financed the execution of this project and the journal's Rapid Service Fee. All authors had full access to the articles reviewed in this manuscript and take complete responsibility for the integrity and accuracy of this manuscript.

Authorship. All named authors meet the International Committee of Medical Journal Editors (ICMJE) criteria for authorship for this article, take responsibility for the integrity of the work as a whole, and have given their approval for this version to be published.

Disclosures. Jose Antonio Pinto Tasende has received payments by conference or scientific consultancies from BMS, Celgene, Janssen, Novartis, Pfizer and MSD. Juan D. Cañete has received fees for advisory boards or grant research from Novartis, Pfizer, Lilly, UCB, Gillead and Celgene. Francisco José Rebollo Laserna and Susana Gómez Castro work for Pfizer S.L.U. Rubén Queiro has nothing to disclose.

Compliance with Ethics Guidelines. This article is based on previously conducted studies and does not contain any studies with human participants or animals performed by any of the authors.

Data Availability. All data generated or analysed during this study are included in this published article/as supplementary information files.

Open Access. This article is licensed under a Creative Commons Attribution-NonCommercial 4.0 International License, which permits any non-commercial use, sharing, adaptation, distribution and reproduction in any medium or format, as long as you give appropriate credit to the original author(s) and the source, provide a link to the Creative Commons licence, and indicate if changes were made. The images or other third party material in this article are included in the article's Creative Commons licence, unless indicated otherwise in a credit line to the material. If material is not included in the article's Creative Commons licence and your intended use is not permitted by statutory regulation or exceeds the permitted use, you will need to obtain permission directly from the copyright holder. To view a copy of this licence, visit http://creativecommons.org/licenses/by$\mathrm{nc} / 4.0 /$.

\section{REFERENCES}

1. Chandran V, Raychaudhuri SP. Geoepidemiology and environmental factors of psoriasis and psoriatic arthritis. J Autoimmun. 2010;34(3):J314-21.

2. Husni ME. Comorbidities in psoriatic arthritis. Rheum Dis Clin North Am. 2015;41(4):677-98.

3. Molto A, Etcheto A, van der Heijde D, et al. Prevalence of comorbidities and evaluation of their screening in spondyloarthritis: results of the international cross-sectional ASAS-COMOSPA study. Ann Rheum Dis. 2016;75(6):1016-23.

4. Queiro R, Lorenzo A, Tejon P, Coto P, Pardo E. Obesity in psoriatic arthritis: Comparative prevalence and associated factors. Medicine (Baltimore). 2019;98(28):e16400.

5. Queiro R, Canete JD. Impact of cardiovascular risk factors on the achievement of therapeutic goals in psoriatic arthritis: is there any association? Clin Rheumatol. 2018;37(3):661-6.

6. Molto A, Dougados M. Comorbidities in spondyloarthritis including psoriatic arthritis. Best Pract Res Clin Rheumatol. 2018;32(3):390-400.

7. Scriffignano S, Perrotta FM, De Socio A, Lubrano E. Role of comorbidities in spondyloarthritis including psoriatic arthritis. Clin Rheumatol. 2019;38(1): $3-10$.

8. Lubrano E, Scriffignano S, De Socio A, Perrotta FM. Analysis of potential determinants for a treat-totarget strategy in psoriatic arthritis patients from a real-world setting. Clin Exp Rheumatol. 2019;37(3): 512. 
9. Brikman S, Furer V, Wollman J, et al. The effect of the presence of fibromyalgia on common clinical disease activity indices in patients with psoriatic arthritis: a cross-sectional study. J Rheumatol. 2016;43(9):1749-54.

10. Di Minno MN, Peluso R, Iervolino S, et al. Weight loss and achievement of minimal disease activity in patients with psoriatic arthritis starting treatment with tumour necrosis factor alpha blockers. Ann Rheum Dis. 2014;73(6):1157-62.

11. Batalla A, Gonzalez-Fernandez D, Gonzalez-Lara L, et al. Cardiovascular risk factors influence response to biological therapies in psoriasis. J Am Acad Dermatol. 2015;73(2):327-9.

12. Miller S, Bawa S. The effect of smoking on treatment response and drug survival in psoriatic arthritis patients treated with their first anti-TNF-a drug: a single-centre retrospective analysis. Ann Rheum Dis. 2013;72.

13. Lupoli R, Pizzicato P, Scalera A, et al. Impact of body weight on the achievement of minimal disease activity in patients with rheumatic diseases: a systematic review and meta-analysis. Arthritis Res Ther. 2016;18(1):297.

14. Husted JA, Thavaneswaran A, Chandran V, Gladman DD. Incremental effects of comorbidity on quality of life in patients with psoriatic arthritis. J Rheumatol. 2013;40(8):1349-56.

15. The Cochrane Collaboration. Cochrane handbook for systematic reviews of interventions. Version 5.1. 0 [updated March 2011]; 2011. https://training. cochrane.org/handbook/archive/v5.1/. Accessed 11 Apr 2019.

16. Shamseer L, Moher D, Clarke M, et al. Preferred reporting items for systematic review and metaanalysis protocols (PRISMA-P) 2015: elaboration and explanation. BMJ. 2015;350:g7647.

17. Centre for Evidence-Based Medicine (CEBM). Medicine; 2011. http://www.cebm.net/index.aspx?o= 1025. Accessed 11 Apr 2013.

18. Abou-Raya A, Abou-Raya S, Helmii M. Effect of exercise and dietary weight loss on symptoms and systemic inflammation in obese adults with psoriatic arthritis: randomized controlled trial. Ann Rheumatic Dis. 2014;73.

19. Aguiar R, Ambrósio C, Cunha I, Barcelos A. Reumasex-sexual satisfaction and factors limiting sexual activity in patients with spondyloarthritis. Ann Rheumatic Dis. 2013;72.

20. Aguiar R, Ambrósio C, Cunha I, Barcelos A. Assessing the sexual impact of rheumatic disease in spondyloarthritis patients-where do we stand? Ann Rheumatic Dis. 2013;72.

21. Akhtari S, Harvey P, Jacobson M, Nagler S, Colaco $\mathrm{K}$, Eder L. A collaborative cardio-rheumatology clinic for primary prevention of cardiovascular diseases-a descriptive study. Arthritis Rheumatol. 2018;70:1271-3.

22. AlJohani R, Polachek A, Ye JY, Chandran V, Gladman DD. Characteristic and outcome of psoriatic arthritis patients with hyperuricemia. J Rheumatol. 2018;45(2):213-7.

23. Arancibia Aguila L, Medeiros AC, Gonc alves CR, Sampaio Barros PD, Goldenstein Schainberg C. Altered sleep patterns, fatigue, anxiety and depression levels are important features among patients with psoriatic arthritis. Ann Rheumatic Dis. 2015;74:1172.

24. Arancibia LA, Gonçalves CR, Sampaio PB, Goldenstein-Schainberg C. Sleep and quality of life in psoriatic arthritis. Clin Exp Rheumatol. 2014;32(5): 795.

25. Bandinelli F, Bartoli F, Bonciani D, et al. Anxiety and depression in early psoriatic arthritis patients. Clin Exp Rheumatol. 2009;27(5):721.

26. Batkaeva N, Batkaev E, Gitinova M. The dermatology life quality index in russian patients with psoriasis and psoriatic arthritis. Acta DermatoVenereol. 2018;98:51.

27. Bessette L, Khraishi M, Florica B, Setty Y, Teo M, Remple V. Impact of extra-articular manifestations on patient-reported outcomes in ankylosing spondylitis and psoriatic arthritis: interim results from the complete studies. Ann Rheum Dis. 2018;77:350-1.

28. Bird P, Griffiths H, Littlejohn G, et al. Fatigue, poor health and mood disturbance persist in rheumatoid arthritis and psoriatic arthritis patients despite disease remission: OPAL Deeper Study [abstract]. Arthritis and Rheumatol. 2017;69[Suppl 10]. https://acrabstracts.org/abstract/fatigue-poor-healthand-mood-disturbance-persist-in-rheumatoid-arthri tis-and-psoriatic-arthritis-patients-despite-diseaseremission-the-opal-deeper-study/.

29. Birra D, Peluso G, Gigante L, et al. BMI does not affect clinical outcome in psoriatic arthritis patients treated with tight control strategy. Ann Rheum Dis. 2018;77:336-7.

30. Cano Garcia L, Hernandez-Sanchez MD, SeguraRuiz R, et al. HPR psychological variables predictive of disorders of sleep in patients with spondylarthritis and psoriatic artrtits. Preliminary multicenter study. Ann Rheum Dis 2017;76:1525. 
31. Cano-Garcia L, Manrique-Arija S, Mena-Vázquez N, et al. Variables predictive of the sleep disorders in patients with psoriatic arthritis and spondylarthritis. Ann Rheum Dis. 2016;75:1291.

32. Garcia LC, Mena-Vázquez N, Hernández-Sánchez $\mathrm{MD}$, et al. HPR psychological variables predictor of sleep disorders in patients with ankilosing spondilylitis and psoriastic artrtits. multicenter study. Ann Rheum Dis. 2018;77:1861.

33. Carneiro SC, Carneiro CS, Chaves M, et al. Fatigue in psoriatic arthritis: correlation between disease activity and various scales (FACIT, PDI, HAQ, BASDAI AND HAD). J Eur Acad Dermatol Venereol. 2010;24:1-2.

34. Castaneda S, Martin-Martinez MA, Gonzalez-Juanatey $\mathrm{C}$, et al. Cardiovascular morbidity and associated risk factors in Spanish patients with chronic inflammatory rheumatic diseases attending rheumatology clinics: baseline data of the CARMA Project. Semin Arthritis Rheum. 2015;44(6):618-26.

35. Cauli A, Gladman DD, Mathieu A, et al. Patient global assessment in psoriatic arthritis: a multicenter GRAPPA and OMERACT study. J Rheumatol. 2011;38(5):898-903.

36. Cetin P, Keser M, Solmaz D, et al. Obesity is associated with psoriatic arthritis and contributes to the increased use of biologics. Ann Rheum Dis. 2015;74:1168.

37. Chimenti MS, Fonti GL, Conigliaro P, et al. Evaluation of alexithymia in patients affected by rheumatoid arthritis and psoriatic arthritis: a crosssectional study. Medicine (Baltimore). 2019;98(4): e13955.

38. Chimenti MS, Triggianese $\mathrm{P}$, Conigliaro $\mathrm{P}$, et al. A 2 -year observational study on treatment targets in psoriatic arthritis patients treated with TNF inhibitors. Clin Rheumatol. 2017;36(10):2253-60.

39. Da Cruz Ribeiro ESE, Da Silva Carneiro SC, Yazbek $\mathrm{MA}$, et al. Correlation of the psoriatic arthritis impact of disease score (PSAID) with the minimal disease activity (MDA) status: a multicenter study. Adv Rheumatol. 2018;58.

40. Dan N, Horia V, Dinu V, et al. The quality of life in patients with secondary osteoporosis due to seronegative spondyloarthropathies. Osteoporos Int. 2011;22:S184-5.

41. De Gaspari CN, Goldeinstein-Schainberg C, Pretti FZ, et al. High rates of work disability in patients with psoriatic arthritis. Ann Rheum Dis. 2015;74:1166.

42. Duffin KC, Wong B, Krueger G. Sleep disturbance and medical comorbidities in patients with psoriasis, psoriatic arthritis, and controls. J Am Acad Dermatol. 2009;60(3):AB9.

43. Duruoz MT, Baklacioglu HS, Sanal Toprak C, Gencer Atalay K, Atagunduz MP. The evaluation of the static and dynamic balance disorders in patients with psoriatic arthritis. Rheumatol Int. 2018;38(11): 2063-8.

44. Duruöz MT, Sari Sürmeli Z, Uçar Ü, Topçu E, Duruöz E. Evaluation of sleep quality in psoriatic arthritis patients. Ann Rheum Dis. 2013;72.

45. Eder L, Thavaneswaran A, Chandran V, Cook RJ, Gladman DD. Obesity is associated with a lower probability of achieving sustained minimal disease activity state among patients with psoriatic arthritis. Ann Rheum Dis. 2015;74(5):813-7.

46. Farkas F, Ikumi N, Szentpetery A, Kirby B, FitzGerald O. Disease activity together with depression contributes to work disability in psoriatic arthritis. Ann Rheum Dis. 2017;76:675.

47. Farkas F, Ikumi N, Szentpetery A, Kirby B, FitzGerald O. Depression and anxiety may contribute to higher disease activity and worse quality of life in psoriatic arthritis. Ann Rheum Dis. 2017;76:1319.

48. Fonti GL, Chimenti MS, Conigliaro P, et al. Investigation of alexithymia in patients affected by rheumatoid and psoriatic arthritis: cross-sectional observation. Ann Rheum Dis. 2018;77:1346.

49. Frede N, Schauer F, Schempp C, et al. Health services research project for the early diagnosis of psoriatic arthritis: monocentric questionnairebased study to identify patients with arthritis and detect signs of depression in 150 patients. Ann Rheum Dis. 2018;77:1594-5.

50. Gezer O, Batmaz I, Sariyildiz MA, et al. Sleep quality in patients with psoriatic arthritis. Clin Exp Rheumatol. 2014;32(5):778.

51. Gossec L, De Wit M, Balanescu A, et al. Patientperceived impact of psoriatic arthritis is due to pain and fatigue, but also to psychological aspects-the PsA impact of disease study. Arthritis Rheum. 2011;63(10).

52. Gottlieb A, Gratacos J, Dikranian A, et al. Treatment patterns, unmet need, and impact on patient-reported outcomes of psoriatic arthritis in the United States and Europe. Rheumatol Int. 2019;39(1): 121-30.

53. Grubišić F, Grazio S, Punda M, Kovačević J, Kusić Z. Morphometric X-ray absorptiometry in the assessment of vertebral fractures in patients with psoriatic arthritis and their relationship to disease activity and functional ability. Ann Rheum Dis. 2013;71. 
54. Gulati AM, Michelsen B, Diamantopoulos A, Grandaunet B, Salvesen O, Kavanaugh A, et al. Osteoporosis in psoriatic arthritis: a cross-sectional study of an outpatient clinic population. RMD Open. 2018;4(1):e000631.

55. Haglund E, Petersson IF, Bremander A, Bergman S. Predictors of work productivity in a population based cohort of individuals with spondyloarthritis. Ann Rheum Dis. 2013;72.

56. Haroon M, Petty-Saphon N, Gallagher P, FitzGerald $\mathrm{O}$. Clinical and patient-reported outcome measures as predictors of poor functional outcome in psoriatic arthritis. Ann Rheum Dis. 2013;72:A682.

57. Huscher D, Albrecht K, Bischoff S, et al. Severe obesity is frequent in patients with psoriatic arthritis and is accompanied by increased cardiovascular risk. Ann Rheum Dis. 2016;75:1152-3.

58. Husted J, Thavaneswaran A, Chandran V, et al. Comparison of comorbid disease burden in psoriasis and psoriatic arthritis (PsA). Arthritis Rheum. 2010;62:1928.

59. Husted JA, Tom BD, Farewell VT, Gladman DD. Longitudinal study of the bidirectional association between pain and depressive symptoms in patients with psoriatic arthritis. Arthritis Care Res (Hoboken). 2012;64(5):758-65.

60. Hyphantis T, Kotsis K, Tsifetaki N, et al. Depressive and anxiety symptoms and illness perceptions associated with physical health-related quality of life in rheumatologic disorders. J Psychosom Res. 2013;74(6):548.

61. Khraishi M, Aslanov R. Prevalence of comorbidities in patients with psoriatic arthritis and their impact on cardiovascular risk and quality of life. Ann Rheum Dis. 2014;73.

62. Kotsis K, Voulgari PV, Tsifetaki N, et al. Anxiety and depressive symptoms and illness perceptions in psoriatic arthritis and associations with physical health-related quality of life. Arthritis Care Res (Hoboken). 2012;64(10):1593-601.

63. Kurizky PS, Mota LM. Sexual dysfunction in patients with psoriasis and psoriatic arthritis-a systematic review. Rev Br Reumatol. 2012;52(6):943-8.

64. Kuru O, Ketenci S, Bilgici A, Alayli G, Akyol Y, Ulus Y. Comparison of sociodemographic characteristics, clinical variables, and depression in patients with psoriasis and psoriatic arthritis. Scand J Rheumatol. 2014;43:66-7.

65. Madeira N, Borges J, Cardoso A, et al. Anxiety and depression on disease activity and quality of life of spondyloarthritis patients under biologic therapies. Ann Rheum Dis. 2017;76:929-30.
66. McDonough E, Thavaneswaran A, Carty A, et al. Depression and anxiety in psoriatic disease: prevalence and associated factors. Arthritis Rheum. 2012;64:S593.

67. Meesters JJL, Petersson IF, Bergman S, Haglund E, Jacobsson LTH, Bremander AB. Factors associated with anxiety and depression in spondyloarthritis patients: Results from the Swedish population based SpA scania cohort. Ann Rheum Dis. 2013;72.

68. Michelsen B, Kristianslund EK, Fageli KM, Lie E, Hammer HB, Haugeberg G, et al. Do depression and anxiety influence the chance of remission in patients with psoriatic arthritis? Real life data from the nor-DMARD study. Ann Rheum Dis. 2017;76: 938-9.

69. Nemes D, Amaricai E, Catan L, et al. Prevalence and differences of the metabolic syndrome in patients with psoriatic arthritis and ankylosing spondylitis. Ann Rheum Dis. 2013;72.

70. Ogdie A, Schwartzman S, Eder L, et al. Comprehensive treatment of psoriatic arthritis: managing comorbidities and extraarticular manifestations. J Rheumatol. 2014;41(11):2315-22.

71. Pistone G, Vitale $\mathrm{P}$, Catanoso $\mathrm{M}$, Macchioni $\mathrm{P}$, Corrao S, Salvarani C. The relationship between visceral adiposity index and disability function in a cohort of psoriatic arthritis patients. Ann Rheum Dis. $2013 ; 72$.

72. Rhee C, Pope J, Thompson A, Le Riche N, Rohekar G, Rohekar S. Comparison of work disability in rheumatoid arthritis, psoriatic arthritis and ankylosing spondylitis patients from a single centre. J Rheumatol. 2012;39(8):1710.

73. Rhee C, Pope JE, Thompson AE, Le Riche NGH, Rohekar G, Rohekar S. Work disability and work limitations in rheumatoid arthritis, psoriatic arthritis and ankylosing spondylitis are equal and increase with comorbidities. Arthritis Rheum. 2012;64:S776.

74. Rhee C, Rohekar S. Assessment of work disability in rheumatoid arthritis, psoriatic arthritis and ankylosing spondylitis. Reumatol Clin Suplement. 2011;7:149.

75. Rosen CF, Mussani F, Chandran V, Eder L, Thavaneswaran A, Gladman DD. Patients with psoriatic arthritis have worse quality of life than those with psoriasis alone. Rheumatology (Oxford). 2012;51(3):571-6.

76. Salaffi F, Carotti M, Gasparini S, Intorcia M, Grassi $\mathrm{W}$. The health-related quality of life in rheumatoid arthritis, ankylosing spondylitis, and psoriatic arthritis: a comparison with a selected sample of 
healthy people. Health Qual Life Outcomes. 2009;7: 25 .

77. Sinnathurai P, Capon A, Buchbinder R, et al. Cardiovascular risk management in rheumatoid and psoriatic arthritis: Online survey results from a national cohort study. BMC Rheumatol. 2018;2(1).

78. Sinnathurai P, Xue M, March L. Obesity and disease activity in psoriatic arthritis: preliminary data from a prospective psoriatic arthritis cohort study. Int Med J. 2018;48:16.

79. Szentpetery A, Ikumi N, Kirby B, Gerald OF. The presence of depression might be an important determinant of achieving minimal disease activity state in psoriatic arthritis. Arthritis Rheumatol. 2016;68:2130-1.

80. Tillett W, Jadon D, Shaddick G, et al. Smoking and delay to diagnosis are associated with poorer functional outcome in psoriatic arthritis. Ann Rheum Dis. 2013;72(8):1358-61.

81. Urruticoechea Arana A, Martin Martinez MA, Castañeda S, et al. Vitamin D deficiency in rheumatoid arthritis, ankylosing spondylitis and psoriatic arthritis. Results of the CARMA study. Ann Rheum Dis. $2015 ; 74: 940$.

82. Vacca A, Porru G, Dessole G, et al. Vitamin D insufficiency and deficiency in two European cohorts of patients with inflammatory rheumatic disorders. Arthritis Rheumatol. 2014;66:S1118-9.

83. Ballegaard C, Hojgaard P, Dreyer L, et al. Impact of comorbidities on tumor necrosis factor inhibitor therapy in psoriatic arthritis: a population-based cohort study. Arthritis Care Res (Hoboken). 2018;70(4):592-9.

84. Bremander A, Jacobsson LT, Bergman S, Haglund E, Lofvendahl S, Petersson IF. Smoking is associated with a worse self-reported health status in patients with psoriatic arthritis: data from a Swedish population-based cohort. Clin Rheumatol. 2015;34(3): 579-83.

85. Carneiro C, Chaves M, Verardino G, et al. Evaluation of fatigue and its correlation with quality of life index, anxiety symptoms, depression and activity of disease in patients with psoriatic arthritis. Clin Cosmetic Investigat Dermatol. 2017;10:155-63.

86. Fernández-Carballido C, Auxiliadora Martin M, García-Gómez MC, et al. The impact of comorbidities on physical function in patients with ankylosing spondylitis and psoriatic arthritis attending rheumatology clinics. Results of a national study. Arthritis Rheumatol 2018;70: $1811-2$
87. Gubar EE, Loginova EY, Koltakova AD, Glukhova SI, Korotaeva TV. Association of anxiety, depression and fatigue with disease activity, joints erosion and skin lesion severity in early psoriatic arthritis patients. Ann Rheum Dis. 2018;77:370.

88. Kennedy M, Papneja A, Thavaneswaran A, Chandran V, Gladman DD. Prevalence and predictors of reduced work productivity in patients with psoriatic arthritis. Clin Exp Rheumatol. 2014;32(3):342-8.

89. Öten E, Başkan B, Sivas F, Bodur H. Relation between osteoporosis and vitamin D levels and disease activity in psoriatic arthritis. Erciyes Med J. 2017;39(3):94-100.

90. Pehlevan S, Yetkin DO, Bahadir C, et al. Increased prevalence of metabolic syndrome in patients with psoriatic arthritis. Metab Syndr Relat Disord. 2014;12(1):43-8.

91. Piche M, Chandran V, Gladman D, Rohekar S. The effect of cigarette smoking and alcohol intake on patient reported outcome measures in psoriatic arthritis. J Rheumatol. 2015;42(7):1321.

92. Sanci P, Kenar G, Zengin B, et al. Metabolic syndrome is associated with active disease in psoriatic arthritis and may contribute to development of syndesmophytes. Ann Rheum Dis. 2018;77:1586.

93. Sandikci SC, Colak S, Aydogan Baykara R, et al. Evaluation of restless legs syndrome and sleep disorders in patients with psoriatic arthritis. Z Rheumatol. 2019;78(10):987-95.

94. Sharma A, Kancharla H, Mishra S, Dogra S. Impact of fibromyalgia on health associated quality of life in patients with psoriatic arthritis. Int J Rheum Dis. 2017;20:5-6.

95. Tanner S, McFadden M, Clegg D, Walsh J. Relationship between psoriatic arthritis severity, duration, and comorbidities. Arthritis Rheumatol. 2014;66:S693-4.

96. Ulutatar F, Unal C, Duruoz MT. Fibromyalgia in patient with psoriatic arthritis: relationship with enthesopathy, sleep, fatigue and quality of life. Ann Rheum Dis. 2018;77:1029-30.

97. Unal C, Duruö MT, Ulutatar F. The relationship between neuropathic pain and disease activity, sleep, fatigue, quality of life in psoriatic arthritis (PsA). J Psoriasis Psoriatic Arthritis. 2018;3(1):32-3.

98. Zaffarana C, Yanzi JG, Cerda OL, Landi M, Schneeberger E, Citera G. Prevalence of obesity in patients with psoriatic arthritis and its impact on the severity of the disease. Arthritis Rheumatol. 2016;68:3715-6. 